\title{
The Likelihood Ranking Methods for Interval Type-2 Fuzzy Sets Considering Risk Preferences
}

\author{
Meng Zhao, ${ }^{1,2}$ Song-song Qin, ${ }^{1,2}$ Qi-wang Li, ${ }^{1,2}$ Fu-qiang Lu, ${ }^{1,2}$ and Zhe Shen ${ }^{2}$ \\ ${ }^{1}$ School of Business Administration, Northeastern University, Shenyang 110819, China \\ ${ }^{2}$ Northeastern University at Qinhuangdao, Qinhuangdao 066004, China \\ Correspondence should be addressed to Meng Zhao; ningmeng5072008@163.com
}

Received 7 April 2015; Revised 10 August 2015; Accepted 19 August 2015

Academic Editor: Dan Simon

Copyright (C) 2015 Meng Zhao et al. This is an open access article distributed under the Creative Commons Attribution License, which permits unrestricted use, distribution, and reproduction in any medium, provided the original work is properly cited.

\begin{abstract}
This paper proposes a ranking method that considers the risk preferences of decision makers for multiple-attribute decision-making problems in a multiple-interval type-2 trapezoidal fuzzy set environment. First, decision makers are classified according to the risk preferences and a measurement method of risk preferences is proposed. Second, a risk preference decision matrix is obtained and a new calculation formula of likelihood is defined. Finally, we obtain the ranking results of alternatives by calculating the signed distance. Our example analysis shows that the proposed method is scientific and reasonable, and different risk preferences influence the results of decision making. Comparison with previous methods shows that the proposed algorithm is more feasible; it is applicable for decision making on both risk preferences and risk conservation.
\end{abstract}

\section{Introduction}

In an increasingly complex decision-making environment, decision-making information has become more uncertain and data with different attributes of options are difficult to determine. In 1965, Zadeh [1] proposed the fuzzy set (type-1 fuzzy set, T1FS) theory that since then has been widely used in multiple-attribute decision-making (MADM) problems. However, the concept of fuzzy sets could not solve the uncertainty of membership, so Zadeh [2] proposed type-2 fuzzy sets (T2FS). This type of fuzzy set is the generalization of the T1FS, describing membership with fuzzy sets in the interval of $[0,1]$. Type-2 fuzzy number is portrayed by the primary and secondary membership. Therefore, T2FS has a stronger ability to deal with uncertain problems. To determine the T2FS, we need to provide an appropriate fuzzy set for membership of each element in the domain, which is difficult. To simplify the problem, we have to impose necessary restrictions on the form of T2FS. One approach is to limit the value of 0 or 1 and obtain the intervalvalue fuzzy sets (equivalent to intuitionistic fuzzy sets and vague set [3]). Another approach is to set the membership function to be the fuzzy number, namely, interval type-2 fuzzy set (IT2FS). IT2FS is a special case of T2FS. The value of the secondary membership is set to 1 and the value of the primary membership is set to a range, thus making it describe uncertainty better than type-1 fuzzy number. IT2FS has a greater application background, especially for MADM problems that require experts to judge the satisfaction degree of options with respect to different attributes. Human cognition has complexity, uncertainty, and other characteristics that cause difficulty for experts to provide a certain value and allows them to provide only linguistic variables that can be represented by fuzzy sets [4]. IT2FS has stronger language explanation ability than ordinary fuzzy sets $[5,6]$. Thus, the MADM problem of IT2FS has become a highly valuable research topic [7].

In 1979, Kahneman and Tversky [8] proposed the significant prospect theory in the field of economics on the basis of a large number of experiments. Kahneman was awarded the Nobel Prize for Economics in 2002 in recognition of his important contribution to economics. Prospect theory states that when decision makers face several decision-making behaviors that have exactly the same amount of theoretical economic benefit, most of the decision makers choose smallrisk decision behaviors. However, some decision makers 
still choose greater-risk decision behaviors. Thus, for uncertain (especially IT2FS) multiattribute group decision-making problems, different risk preferences directly influence the decision results. Therefore, we need to classify decision makers in accordance with their risk preference before entering the specific decision-making process. However, existing studies generally do not consider the risk preference or assume risk aversion, so no effective approach is available for IT2FS multiattribute decision problems.

Considering this situation, we propose the risk preference measurement method of IT2TrFS. First, we classify decision makers on the basis of different risk preferences and obtain a risk preference decision matrix for decision makers. Second, we propose a new calculation method of likelihood. Finally, we obtain the ranking results of options by calculating the signed distance. This method takes into account the risk preferences of decision makers and proposes a specific decision method for IT2TrFS MADM problems with attribute weights in the form of an exact value of IT2TrFS.

\section{Literature Review}

At present, the theoretical study of interval type-2 fuzzy sets (IT2FS) mainly focuses on pure mathematics. For example, Chen [9] studied the nature and operation of IT2FS, Zheng et al. [10] analyzed the similarity and acceptance of IT2FS, Zarinbal et al. [11] proposed a clustering analysis model of IT2FS with the method of relative entropy, Hwang et al. [12] proposed a similarity measurement method of interval type2 fuzzy entropy, and Li et al. [13] proposed the uncertainty measurement method of IT2FS. These theoretical studies serve as a good theoretical foundation for the research on IT2FS in the field of multiattribute decision making (MADM).

However, to solve IT2FS MADM problems, we need to address the translation problem between IT2FS and linguistics. In recent years, research on the translation between IT2FS and linguistics mainly includes three-level [14], fourlevel [15], five-level [16], seven-level [17, 18], and ninelevel $[19,20]$ languages. Using these language-level systems, decision makers can easily convert linguistic variables into IT2FS to make decisions.

IT2FS mainly includes IT2IFS, interval type-2 triangular fuzzy sets (IT2TFS), interval type-2 trapezoidal fuzzy sets (IT2TrFS), and others. The IT2IFS domain is a discrete set and can only roughly indicate whether an attribute is the member of an option or not. Therefore, conducting research based on IT2TFS and IT2TrFS is more meaningful than research on IT2IFS. Previous studies on IT2TFS MADM are as follows. Mokhtarian et al. [21] proposed IT2TFS MADM of controlling risk. Using IT2TFS decision problems, Ashtiani et al. [22] presented a feasible method to solve multiattribute group decision-making problems with the improved TOPSIS algorithm. Guo and Yin [23] proposed type-2 intuitionistic fuzzy information MADM methods. Compared to IT2TrFS, IT2TFS can describe the qualitative index [7, 24, 25] more effectively. Studies on IT2TrFS have started only recently.
For example, Chen et al. [7, 24, 25] improved the classic QUALIFLEX, ELECTRE, and PROMETHEE algorithms and proposed a specific MADM algorithm. Xu et al. [26] presented a conflict measurement model based on interval intuitionistic fuzzy number preference for large group decision-making problems. Zamri and Abdullah [27] proposed IT2TrFS entropy MADM methods. These studies provided specific ideas and laid a good foundation for our study. However, existing studies do not consider the impact of risk preferences of decision makers on decision results. For fuzzy MADM problems, decision makers have to be classified in accordance with their risk preferences. As IT2FS is used to portray ambiguity and uncertainty, introducing the risk preferences of decision makers in making decisions is a practical approach [28-30].

\section{Preliminary Knowledge}

Let $X$ be a crisp set. Let $\operatorname{Int}([0,1])$ denote the set of all closed subintervals of $[0,1]$. A mapping $A: X \rightarrow X([0,1])$ is known as an IT2FS in $X$. For each $x \in X, A(x)=\left[A^{-}(x), A^{+}(x)\right] \subseteq$ $[0,1]$ represents the degree of membership of an element $x$ to $A$. The type- 1 fuzzy sets $A^{-}: X \rightarrow[0,1]$ and $A^{+}: X \rightarrow[0,1]$ are referred to as a lower fuzzy set of $A$ and an upper fuzzy set of $A$, respectively. The values $A^{-}(x)$ and $A^{+}(x)$ represent the degrees of membership of $x \in X$ to $A^{-}$and $A^{+}$, respectively, where $0 \leq A^{-}(x) \leq A^{+}(x) \leq 1$. If $A^{-}(x)=A^{+}(x)$ for all $x \in X$, then the IT2FS $A$ is fully determined by a single value from $[0,1]$ for each $x$, and $A$ reduces to a T1FS.

Let $a_{1}{ }^{-}, a_{2}{ }^{-}, a_{3}{ }^{-}, a_{4}{ }^{-}, a_{1}{ }^{+}, a_{2}{ }^{+}, a_{3}{ }^{+}$, and $a_{4}{ }^{+}$be nonnegative real values, where $0 \leq a_{1}{ }^{-} \leq a_{2}{ }^{-} \leq a_{3}{ }^{-} \leq a_{4}{ }^{-}, 0 \leq a_{1}{ }^{+} \leq$ $a_{2}{ }^{+} \leq a_{3}{ }^{+} \leq a_{4}{ }^{+}, a_{1}{ }^{+} \leq a_{1}{ }^{-}$, and $a_{4}{ }^{-} \leq a_{4}{ }^{+}$. Let $h_{A}{ }^{-}$ and $h_{A}{ }^{+}$denote the heights of $A^{-}$and $A^{+}$, respectively, where $0 \leq h_{A}{ }^{-} \leq h_{A}{ }^{+} \leq 1$. We consider that the lower and upper membership functions $A^{-}(x)$ and $A^{+}(x)$, respectively, of $A$ are defined as follows:

$$
\begin{aligned}
& A^{-}(x)= \begin{cases}\frac{h_{A}{ }^{-}\left(x-a_{1}{ }^{-}\right)}{a_{2}{ }^{-}-a_{1}{ }^{-}} & a_{1}{ }^{-}<x<a_{2}{ }^{-}, \\
h_{A}{ }^{-} & a_{2}{ }^{-} \leq x \leq a_{3}{ }^{-}, \\
\frac{h_{A}{ }^{-}\left(a_{4}{ }^{-}-x\right)}{a_{4}{ }^{-}-a_{3}{ }^{-}} & a_{3}{ }^{-}<x<{a_{4}}^{-}, \\
0 & \text { otherwise; }\end{cases} \\
& A^{+}(x)= \begin{cases}\frac{h_{A}{ }^{+}\left(x-a_{1}{ }^{+}\right)}{a_{2}{ }^{+}-a_{1}{ }^{+}} & a_{1}{ }^{+}<x<a_{2}{ }^{+}, \\
h_{A}{ }^{+} & a_{2}{ }^{+} \leq x \leq a_{3}{ }^{+}, \\
\frac{h_{A}{ }^{+}\left(a_{4}{ }^{+}-x\right)}{a_{4}{ }^{+}-a_{3}{ }^{+}} & a_{3}{ }^{+}<x<a_{4}{ }^{+}, \\
0 & \text { otherwise. }\end{cases}
\end{aligned}
$$

The lower and upper nonnegative trapezoidal fuzzy numbers with respect to $A$ are denoted by $A^{-}=$ $\left(a_{1}{ }^{-}, a_{2}{ }^{-}, a_{3}{ }^{-}, a_{4}{ }^{-} ; h_{A}{ }^{-}\right)$and $A^{+}=\left(a_{1}{ }^{+}, a_{2}{ }^{+}, a_{3}{ }^{+}, a_{4}{ }^{+} ; h_{A}{ }^{+}\right)$, respectively, where $A^{-} \subseteq A^{+}$(i.e., if and only if, $\forall x \in X$, 


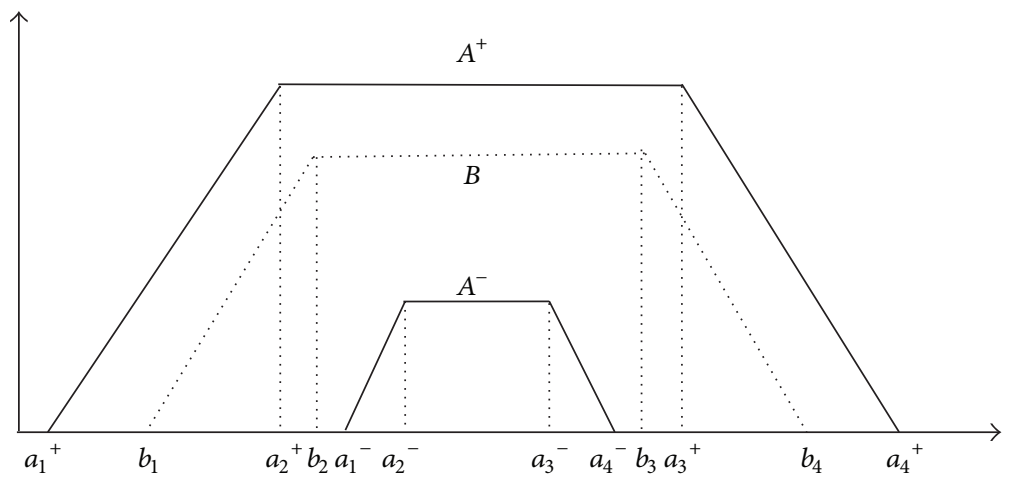

FIgURE 1: Measurement of IT2TrF risk preferences.

$\left.A^{-}(x) \leq A^{+}(x)\right)$. Additionally, $A$ is a nonnegative IT2TrF number in $X$ and is expressed as follows:

$$
\begin{aligned}
& A=\left[A^{-}, A^{+}\right] \\
& =\left[\left(a_{1}{ }^{-}, a_{2}{ }^{-}, a_{3}{ }^{-}, a_{4}{ }^{-} ; h_{A}{ }^{-}\right),\left(a_{1}{ }^{+}, a_{2}{ }^{+}, a_{3}{ }^{+}, a_{4}{ }^{+} ; h_{A}{ }^{+}\right)\right] .
\end{aligned}
$$

By applying the extension principle proposed by Zadeh [31] to the IT2TrF environment, the operations of addition and multiplication by a nonnegative ordinary number defined on nonnegative IT2TrF numbers produce another nonnegative IT2TrF number. Let $A_{\rho}$ and $A_{\beta}$ be two nonnegative IT2TrF numbers in $X$, where

$$
\begin{gathered}
A_{\rho}=\left[\left(a_{1 \rho}{ }^{-}, a_{2 \rho}{ }^{-}, a_{3 \rho}{ }^{-}, a_{4 \rho}{ }^{-} ; h_{A \rho}{ }^{-}\right),\right. \\
\left.\left(a_{1 \rho}{ }^{+}, a_{2 \rho}{ }^{+}, a_{3 \rho}{ }^{+}, a_{4 \rho}{ }^{+} ; h_{A \rho}{ }^{+}\right)\right], \\
A_{\beta}=\left[\left({a_{1 \beta}}^{-}, a_{2 \beta}{ }^{-}, a_{3 \beta}{ }^{-}, a_{4 \beta}{ }^{-} ; h_{A \beta}{ }^{-}\right),\right. \\
\left.\left(a_{1 \beta}{ }^{+}, a_{2 \beta}{ }^{+}, a_{3 \beta}{ }^{+}, a_{4 \beta}{ }^{+} ; h_{A \beta}{ }^{+}\right)\right] .
\end{gathered}
$$

The addition operation between $A_{\rho}$ and $A_{\beta}$ is defined as follows:

$$
\begin{aligned}
A_{\rho} & \oplus A_{\beta}=\left[\left(a_{1 \rho}{ }^{-}+a_{1 \beta}{ }^{-}, a_{2 \rho}{ }^{-}+a_{2 \beta}{ }^{-}, a_{3 \rho}{ }^{-}\right.\right. \\
& \left.+a_{3 \beta}{ }^{-}, a_{4 \rho}{ }^{-}+a_{4 \beta}{ }^{-} ; \min \left\{h_{A \rho}{ }^{-}, h_{A \beta}{ }^{-}\right\}\right),\left(a_{1 \rho}{ }^{+}\right. \\
& +a_{1 \beta}{ }^{+}, a_{2 \rho}{ }^{+}+a_{2 \beta}{ }^{+}, a_{3 \rho}{ }^{+}+a_{3 \beta}{ }^{+}, a_{4 \rho}{ }^{+} \\
& \left.\left.+a_{4 \beta}{ }^{+} ; \min \left\{h_{A \rho}{ }^{+}, h_{A \beta}{ }^{+}\right\}\right)\right] .
\end{aligned}
$$

The multiplication operation between a nonnegative real value $\lambda$ and $A_{\rho}$ is defined as follows:

$$
\begin{gathered}
\lambda \cdot A_{\rho}=\left[\left(\lambda \cdot{a_{1 \rho}}^{-}, \lambda \cdot a_{2 \rho}{ }^{-}, \lambda \cdot{a_{3 \rho}}^{-}, \lambda \cdot{a_{4 \rho}}^{-} ; h_{A \rho}{ }^{-}\right),\right. \\
\left.\left(\lambda \cdot{a_{1 \rho}}^{+}, \lambda \cdot{a_{2 \rho}}^{+}, \lambda \cdot{a_{3 \rho}}^{+}, \lambda \cdot{a_{4 \rho}}^{+} ; h_{A \rho}{ }^{+}\right)\right] .
\end{gathered}
$$

\section{Theoretical Basis}

In this paper, the proposed IT2IFS MADM method considering risk preference mainly refers to two core contents.
One aspect is measuring the risk appetite of IT2IFS makers. Another is choosing the suitable ranking method. The two aspects are explained in Sections 4.1 and 4.2.

\subsection{Measurement of Risk Preferences of Decision Makers}

4.1.1. Fundamental Principle. Each IT2TrF has a lower limit and upper limit simultaneously. For example, according to Figure 1, the lower limit and upper limit of IT2 $\operatorname{TrF} A$ are $A^{-}=$ $\left(a_{1}{ }^{-}, a_{2}{ }^{-}, a_{3}{ }^{-}, a_{4}{ }^{-} ; h_{A}{ }^{-}\right)$and $A^{+}=\left(a_{1}{ }^{+}, a_{2}{ }^{+}, a_{3}{ }^{+}, a_{4}{ }^{+} ; h_{A}{ }^{+}\right)$, respectively. These lower and upper limits are type-1 trapezoidal fuzzy numbers. The area between the lower and upper limits is the fuzzy zone of an IT2TrF; namely, IT2TrF is the set of all type-1 trapezoidal fuzzy numbers in a fuzzy field. The core of risk preference measurement of decision makers lies in different attitudes toward the fuzzy zone. When the decision that a decision maker makes is closer to $A^{-}$, the risk preference is close to 0 and the decision maker belongs to the type of complete risk avoidance. When the decision is closer to $A^{+}$, the risk preference is close to 1 and the decision maker belongs to the type of complete risk preference. Based on the different risk preferences of decision makers, the value of decision making is between $A^{-}$and $A^{+}$. Therefore, the value of risk preference of decision makers is also between 0 and 1 . According to this principle, a risk preference coefficient $\theta$ of decision makers is introduced.

4.1.2. Measurement Method. According to the basic principle of risk preference measurement of decision makers and Figure 1, we propose the following risk preference measurement formula:

(1) When $a_{1}{ }^{+}<a_{2}{ }^{+}<a_{1}{ }^{-}<a_{2}{ }^{-}<a_{3}{ }^{-}<a_{4}{ }^{-}<a_{3}{ }^{+}<$ $a_{4}{ }^{+}$,

$\theta$

$=\frac{\left[\left(b_{4}-b_{1}+b_{3}-b_{2}\right) h_{B}\right]-\left[\left(a_{4}{ }^{-}-a_{1}{ }^{-}+a_{3}{ }^{-}-a_{2}{ }^{-}\right) h_{A^{-}}\right]}{\left[\left(a_{4}{ }^{+}-a_{1}{ }^{+}+a_{3}{ }^{+}-a_{2}{ }^{+}\right) h_{A^{+}}\right]-\left[\left(a_{4}^{-}-a_{1}{ }^{-}+a_{3}{ }^{-}-a_{2}{ }^{-}\right) h_{A^{-}}\right]}$.

(2) When $\left(a_{1}{ }^{+}=a_{2}{ }^{+}\right) \wedge\left(a_{1}{ }^{-}=a_{2}{ }^{-}\right) \wedge\left(a_{3}{ }^{-}=a_{4}{ }^{-}\right)<$ $\left(a_{3}^{+}=a_{4}^{+}\right)$,

$$
\theta=0 .
$$


(3) When $a_{1}{ }^{+}=a_{2}{ }^{+}=a_{1}{ }^{-}=a_{2}{ }^{-}=a_{3}{ }^{-}=a_{4}{ }^{-}=a_{3}{ }^{+}=$ $a_{4}^{+}$,

$$
\theta=0
$$

According to formula (7)-(9), the range of risk preference coefficient is $0 \leq \theta \leq 1$. With increasing $\theta$, the decision maker is inclined toward risk preference. The given calculation method of risk preference coefficient is based on the proportional relationship of the trapezoid area. The trapezoid area of the decision making is determined by subjective risk preference, in this sense that risk preference coefficient is computed subjectively.

4.2. Likelihood of Trapezoidal Fuzzy Number Preference Relations. The ranking method based on likelihood is one of the most widely used ranking methods for FMADM [30], and it has many excellent properties such as transmission and complementation. Particularly for interval type-2, Chen [25] proved through experimental studies that the ranking method based on likelihood is more convenient to calculate and more practical than other methods such as QUALIFLEX, ELECTRE, and TOPSIS. However, Chen did not consider the risk preferences of decision makers in his interval type- 2 set ranking method based on likelihood. Considering that Chen's study does not apply definition of likelihood, we provide the new definition of likelihood of trapezoidal fuzzy number preference relations.

Assuming two nonnegative trapezoidal fuzzy numbers $A_{\rho}$ and $A_{\beta}$ in $X$, preference relation $A_{\rho} \geq A_{\beta}$ denotes that $A_{\rho}$ is better than or equal to $A_{\beta}$. When preference relation $A_{\rho} \geq A_{\beta}$ holds, $L\left(A_{\rho} \geq A_{\beta}\right)$ is defined as the likelihood between trapezoidal fuzzy numbers.

With respect to the two nonnegative trapezoidal fuzzy numbers $A_{\rho}=\left(a_{1 \rho}, a_{2 \rho}, a_{3 \rho}, a_{4 \rho} ; h_{A \rho}\right)$ and $A_{\beta}=\left(a_{1 \beta}, a_{2 \beta}, a_{3 \beta}, a_{4 \beta} ; h_{A \beta}\right)$ in $X$, we assume that at least one of $h_{A \rho} \neq h_{A \beta}$ and $a_{\xi \rho}{ }^{-} \neq a_{\xi \beta}{ }^{+}(\xi=1,2,3,4)$ holds. Then, the likelihood $L\left(A_{\rho} \geq A_{\beta}\right)$ between trapezoidal fuzzy numbers is defined as follows:

$$
L\left(A_{\rho} \geq A_{\beta}\right)=\max \left\{\min \left[1-\frac{\sum_{\xi=1}^{4} \max \left(a_{\xi \beta}-a_{\xi \rho}, 0\right)+\left(a_{4 \beta}-a_{1 \rho}\right)+2 \max \left(h_{A \beta}-h_{A \rho}, 0\right)}{\sum_{\xi=1}^{4}\left|a_{\xi \beta}-a_{\xi \rho}\right|+\left(a_{4 \rho}-a_{1 \rho}\right)+\left(a_{4 \beta}-a_{1 \beta}\right)+2\left|h_{A \beta}-h_{A \rho}\right|}, 1\right]\right\}
$$

The properties of likelihood $L\left(A_{\rho} \geq A_{\beta}\right)$ are the following (Appendix B provides a demonstration):

(1) $0 \leq L\left(A_{\rho} \geq A_{\beta}\right) \leq 1$;

(2) $L\left(A_{\rho} \geq A_{\beta}\right)+L\left(A_{\beta} \geq A_{\rho}\right)=1$;

(3) If $L\left(A_{\rho} \geq A_{\beta}\right)=L\left(A_{\beta} \geq A_{\rho}\right), L\left(A_{\rho} \geq A_{\beta}\right)=L\left(A_{\beta} \geq\right.$ $\left.A_{\rho}\right)=0.5$

(4) $L\left(A_{\rho} \geq A_{\rho}\right)=0.5$.

\section{Model Algorithm}

5.1. Problem. We consider an MCDA problem of IT2TrF in which the ratings of the alternative evaluations and criterion importance are expressed as IT2TrF numbers. We define $Z=$ $\left\{z_{1}, z_{2}, \ldots, z_{m}\right\}$ as a set of decision alternatives, where $m$ is the number of alternatives. We define $C=\left\{c_{1}, c_{2}, \ldots, c_{n}\right\}$ as a criteria set that contains the criteria by which the alternative performances are measured and where $n$ is the number of criteria. The set $C$ can generally be divided into two sets, $C_{\mathrm{I}}$ and $C_{\mathrm{II}}\left(C_{\mathrm{I}} \cap C_{\mathrm{II}}=\emptyset, C_{\mathrm{I}} \cup C_{\mathrm{II}}=C\right)$, where $C_{\mathrm{I}}$ denotes a collection of benefit criteria (i.e., a larger value indicates greater preference) and $C_{\mathrm{II}}$ denotes a collection of cost criteria (i.e., a smaller value indicates greater preference).

Let a nonnegative IT2TrF number $A_{i j}$ denote the evaluative rating of an alternative $z_{i} \in Z$ with respect to a criterion $c_{j} \in C$. Let $A_{i}{ }^{-}\left(x_{j}\right)$ and ${A_{i}}^{+}\left(x_{j}\right)$ denote the lower and upper membership functions of $A_{i j}$, respectively, where $A_{i j}$ is represented as follows:

$$
\begin{gathered}
A_{i j}=\left[A_{i j}{ }^{-}, A_{i j}{ }^{+}\right]=\left[\left(a_{1 i j}{ }^{-}, a_{2 i j}{ }^{-}, a_{3 i j}{ }^{-}, a_{4 i j}{ }^{-} ; h_{A j}{ }^{-}\right),\right. \\
\left.\left({a_{1 i j}}^{+},{a_{2 i j}}^{+},{a_{3 i j}}^{+},{a_{4 i j}}^{+} ; h_{A j}{ }^{+}\right)\right],
\end{gathered}
$$

where $0 \leq a_{1 i j}{ }^{-} \leq a_{2 i j}{ }^{-} \leq a_{3 i j}{ }^{-} \leq a_{4 i j}{ }^{-}, 0 \leq a_{1 i j}{ }^{+} \leq a_{2 i j}{ }^{+} \leq$ $a_{3 i j}{ }^{+} \leq a_{4 i j}{ }^{+}, a_{1 i j}{ }^{+} \leq a_{1 i j}{ }^{-}, a_{4 i j}{ }^{-} \leq{a_{4 i j}}^{+}, 0 \leq h_{A i j}{ }^{-} \leq h_{A i j}{ }^{+} \leq 1$, and $A_{i j}{ }^{-} \subseteq A_{i j}{ }^{+}$(i.e., if and only if, $\forall x_{j} \in X, A_{i}{ }^{-}\left(x_{j}\right) \leq$ $\left.A_{i}{ }^{+}\left(x_{j}\right)\right)$.

Similarly, let a nonnegative IT2TrF number $W_{j}$ denote the importance weight of a criterion $c_{j} \in C$. Let $W^{-}\left(x_{j}\right)$ and $W^{+}\left(x_{j}\right)$ denote the lower and upper membership functions of $W_{j}$, respectively. $W_{j}$ is expressed as follows:

$$
\begin{gathered}
W_{j}=\left[W_{j}^{-}, W_{j}^{+}\right]=\left[\left(W_{1 j}{ }^{-}, W_{2 j}^{-}, W_{3 j}^{-}, W_{4 j}{ }^{-} ; h_{W j}{ }^{-}\right),\right. \\
\left.\left({W_{1 j}}^{+},{W_{2 j}}^{+}, W_{3 j}{ }^{+}, W_{4 j}{ }^{+} ; h_{W j}{ }^{+}\right)\right],
\end{gathered}
$$

where $0 \leq W_{1 j}{ }^{-} \leq W_{2 j}{ }^{-} \leq W_{3 j}{ }^{-} \leq W_{4 j}{ }^{-}, 0 \leq W_{1 j}{ }^{+} \leq W_{2 j}{ }^{+} \leq$ $W_{3 j}{ }^{+} \leq W_{4 j}{ }^{+}, W_{1 j}{ }^{+} \leq W_{1 j}{ }^{-}, W_{4 j}{ }^{-} \leq W_{4 j}{ }^{+}, 0 \leq h_{W j}{ }^{-} \leq h_{W j}{ }^{+} \leq$ 1 , and $W_{j}^{-} \subseteq W_{j}^{+}$(i.e., if and only if, $\forall x_{j} \in X, W^{-}\left(x_{j}\right) \leq$ $\left.W^{+}\left(x_{j}\right)\right)$.

Based on the approach of converting linguistic variables to IT2TrF numbers, where the importance weights of each attribute and the evaluative ratings of each alternative 
TABLE 1: Risk preference and corresponding risk preference coefficient $\theta$.

\begin{tabular}{lc}
\hline Risk preference & Risk preference coefficient $\theta$ \\
\hline Absolute risk aversion & 0 \\
Very strong risk aversion & 0.1 \\
Strong risk aversion & 0.2 \\
Medium risk aversion & 0.3 \\
Weak risk aversion & 0.4 \\
Risk neutral & 0.5 \\
Weak risk preference & 0.6 \\
Medium risk preference & 0.7 \\
Strong risk preference & 0.8 \\
Very strong risk preference & 0.9 \\
Absolute risk preference & 1 \\
\hline
\end{tabular}

are converted to IT2TrF numbers, the following proposed algorithm is proposed to solve the problem of obtaining the different ranking results with different risk preference coefficient $\theta$. Thus, decision makers can select risk preference coefficient $\theta$ according to the environment to rank the alternatives (see Table 1).

5.2. Proposed Algorithm. The proposed IT2TrF MADM considering risk preference is as follows.

(1) The Decision Matrix A Is Initialized. An MCDA problem is formulated. The alternative set $Z=\left\{z_{1}, z_{2}, \ldots, z_{m}\right\}$ and the criterion set $C=\left\{c_{1}, c_{2}, \ldots, c_{n}\right\}$ are specified. The decision matrix $A$ is established. $A_{i j}$ denotes the evaluation value of alternative $z_{i}$ with respect to the criterion $c_{j}$. Matrix $A$ is expressed as follows:

$$
A=\left[\begin{array}{cccc}
A_{11} & A_{12} & \cdots & A_{1 n} \\
A_{21} & A_{22} & \cdots & A_{2 n} \\
\vdots & \vdots & & \vdots \\
A_{m 1} & A_{m 2} & \cdots & A_{m n}
\end{array}\right]
$$

(2) The Risk Preference Coefficient $\theta$ Is Introduced. To differentiate the risk preferences of different decision makers, we introduce risk preference coefficient $\theta$ and translate IT2TrF $A=\left[A^{-}, A^{+}\right]$into interval type-1 trapezoidal fuzzy number $B=\left[b_{1}, b_{2}, b_{3}, b_{4} ; h_{b}\right]$, shown in the following formula:

$$
\begin{aligned}
& B=\theta A^{-}+(1-\theta) A^{+} \\
& B=\left[\begin{array}{cccc}
B_{11} & B_{12} & \cdots & B_{1 n} \\
B_{21} & B_{22} & \cdots & B_{2 n} \\
\vdots & \vdots & & \vdots \\
B_{m 1} & B_{m 2} & \cdots & B_{m n}
\end{array}\right],
\end{aligned}
$$

where $B_{i j}=\left(b_{1 i j}, a_{2 i j}, a_{3 i j}, a_{4 i j} ; h_{i j}\right)$.
(3) Likelihood Computation $L\left(B_{i j} \geq B_{i^{\prime} j}\right)$ Is Performed. Formula (9) is applied to compute the likelihoods $L\left(B_{i j} \geq\right.$ $B_{i^{\prime} j}$ ) for each criterion $c_{j} \in C$ and each pair of alternatives $z_{i} \in Z$ and $z_{i^{\prime}} \in Z\left(i^{\prime}=1,2, \ldots, m, i^{\prime} \neq i\right)$.

(4) The Likelihood-Based Performance Index $P\left(B_{i j}\right)$ Is Calculated. The likelihood $L\left(B_{i j} \geq B_{i^{\prime} j}\right)$ represents the possibility of $B_{i j} \geq B_{i^{\prime} j}$ holding. If the evaluative rating $B_{i j}$ has a high possibility of being greater than or equal to the evaluative rating $B_{i^{\prime} j}$, then the alternative $z_{i} \in Z$ results in improved performance for a benefit criterion $c_{j} \in C_{\mathrm{I}}$ compared to $z_{i^{\prime}} \notin Z$. By contrast, if the evaluative rating $B_{i^{\prime} j}$ has a high possibility of being greater than or equal to the evaluative rating $B_{i j}$, the alternative $z_{i^{\prime}} \notin Z$ results in improved performance for a benefit criterion $c_{j} \in C_{\mathrm{II}}$ compared to $z_{i} \in Z . P\left(B_{i j}\right)$ is defined as follows:

$$
P\left(B_{i j}\right)= \begin{cases}\sum_{i^{\prime}=1, i^{\prime} \neq i}^{m} L\left(B_{i j} \geq B_{i^{\prime} j}\right), & c_{j} \in C_{\mathrm{I}}, \\ \sum_{i^{\prime}=1, i^{\prime} \neq i}^{m} L\left(B_{i^{\prime} j} \geq B_{i j}\right), & c_{j} \in C_{\mathrm{II}} .\end{cases}
$$

(5) The Comprehensive Evaluation Value of Each Alternative $E_{i}$ Is Calculated. In MADM problems, different attributes are usually given different weights. Considering the impact of attribute weights on the basis of the evaluation $P\left(B_{i j}\right)$, we introduce a comprehensive evaluation $E_{i}$ of $z_{i} \in Z$ defined as follows:

$$
\begin{aligned}
E_{i} & =\bigoplus_{j=1}^{n} P\left(B_{i j}\right) \cdot W_{j}=\left[\left(\sum_{j=1}^{n} P\left(B_{i j}\right)\right.\right. \\
& \cdot W_{1 j}{ }^{-}, \sum_{j=1}^{n} P\left(B_{i j}\right) \cdot W_{2 j}{ }^{-}, \sum_{j=1}^{n} P\left(B_{i j}\right) \\
& \left.\cdot W_{3 j}{ }^{-}, \sum_{j=1}^{n} P\left(B_{i j}\right) \cdot W_{4 j}{ }^{-} ; \min _{j=1}^{n} h_{W j}{ }^{-}\right),\left(\sum_{j=1}^{n} P\left(B_{i j}\right)\right. \\
& \cdot W_{1 j}{ }^{+}, \sum_{j=1}^{n} P\left(B_{i j}\right) \cdot W_{2 j}{ }^{+}, \sum_{j=1}^{n} P\left(B_{i j}\right) \\
& \left.\left.\cdot W_{3 j}{ }^{+}, \sum_{j=1}^{n} P\left(B_{i j}\right) \cdot W_{4 j}{ }^{+} ; \min _{j=1}^{n} h_{W j}{ }^{+}\right)\right] \\
E_{i} & =\left[E_{i}^{-}, E_{i}^{+}\right]=\left[\left(e_{1 i}{ }^{-}, e_{2 i}{ }^{-}, e_{3 i}^{-}, e_{4 i}{ }^{-} ; h_{E i}{ }^{-}\right),\right. \\
& \left.\left(e_{1 i}{ }^{+}, e_{2 i}{ }^{+}, e_{3 i}{ }^{+}, e_{4 i}{ }^{+} ; h_{E i}{ }^{+}\right)\right],{ }^{-}
\end{aligned}
$$

where $0 \leq e_{1 i}{ }^{-} \leq e_{2 i}{ }^{-} \leq e_{3 i}{ }^{-} \leq e_{4 i}{ }^{-}, 0 \leq e_{1 i}{ }^{+} \leq e_{2 i}{ }^{+} \leq$ $e_{3 i}{ }^{+} \leq e_{4 i}{ }^{+}, e_{1 i}{ }^{+} \leq e_{1 i}{ }^{-}, e_{4 i}{ }^{-} \leq e_{4 i}{ }^{+}$, and $0 \leq h_{E i}{ }^{-} \leq h_{E i}{ }^{+} \leq 1$, $E_{i}^{-} \subseteq E_{i}^{+}$. 
(6) Alternatives Are Ranked. Comprehensive evaluation $E_{i}$ is IT2 $\operatorname{TrF}$, so $E_{i}$ cannot be directly compared with each other. Therefore, to rank the comprehensive evaluation $E_{i}(i=$ $1,2, \ldots, m)$, we define the signed distance [24] $\varepsilon_{i}$ as follows:

$$
\begin{aligned}
\varepsilon_{i}= & \frac{1}{8}\left[e_{1 i}^{-}+e_{2 i}^{-}+e_{3 i}{ }^{-}+e_{4 i}^{-}+4 \cdot e_{1 i}{ }^{+}+2 e_{2 i}{ }^{+}\right. \\
& +2 e_{3 i}^{+}+4 e_{4 i}^{+} \\
& \left.+3\left(e_{2 i}^{+}+e_{3 i}^{+}-e_{1 i}^{+}-e_{4 i}^{+}\right) \frac{h_{E_{i}}{ }^{-}}{{h_{E_{i}}}^{+}}\right]
\end{aligned}
$$

$\varepsilon_{i}$ denotes the distance of comprehensive evaluation index $E_{i}$

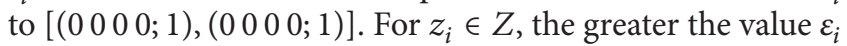
is, the better the alternative is than others.

\section{Applications and Comparative Discussions}

6.1. Application to Supplier Selection Problem. An aviation project needs to select one supplier from four candidate enterprises. The supplier charges the production of an aircraft wing-body fairing. Nine attributes can be based on facilities assurance $\left(c_{1}\right)$, technological compatibility $\left(c_{2}\right)$, degree of complementarity $\left(c_{3}\right)$, win-win nature $\left(c_{4}\right)$, management experience $\left(c_{5}\right)$, collaborative degree $\left(c_{6}\right)$, credibility $\left(c_{7}\right)$, prospects $\left(c_{8}\right)$, and innovation $\left(c_{9}\right)$; namely, the attribute set $C=\left(c_{1}, c_{2}, c_{3}, \ldots, c_{9}\right)$. Chen et al. [7] used a nine-point linguistic rating scale (i.e., absolutely poor, very poor, poor, medium poor, fair, medium good, good, very good, and absolutely good) to evaluate the options. Higher linguistic rating values indicate greater preference; thus, all of the criteria in $C$ can be considered as benefit criteria, and $C_{\mathrm{I}}=C$ and $C_{\mathrm{II}}=\phi$. Based on the approach of converting linguistic variables to IT2TrF numbers, the importance weight $\omega_{j}$ of each $c_{j}$ and the evaluative ratings of each $z_{i}$ with respect to $c_{j}$ are established and presented in Tables 5 to 6 .

In the present study, we introduce risk preference coefficient $\theta$ for different risk preferences. Using the proposed method for IT2TrFS MCDA, we obtain different ranking results of alternatives with different risk preference coefficients, which are presented in Table 2.

As shown in Table 2, when decision makers are inclined to risk aversion, namely, $\theta \in[0,0.4]$, the best treatment option is $Z_{1}$; and when they are inclined to risk preference and neutral, namely, $\theta \in[0.5,1.0]$, the best treatment option is $Z_{2}$. In the case of the aviation project, the decision makers are risk averse and the company is on a steady growth path; thus, it needs to make sound decisions. The risk preference coefficient of decision makers is 0.2 , so the best selection is program $Z_{1}$.

6.2. Application to Engineering Investment Project Evaluation Problem. To solve the engineering investment project
TABLE 2: The ranking results with different $\theta$.

\begin{tabular}{lcccl}
\hline & $\varepsilon_{1}$ & $\varepsilon_{2}$ & $\varepsilon_{3}$ & Ranking results \\
\hline$\theta=0$ & 11.2168 & 11.1865 & 10.5695 & $z_{1}>z_{2}>z_{3}$ \\
$\theta=0.1$ & 11.2056 & 11.1770 & 10.5903 & $z_{1}>z_{2}>z_{3}$ \\
$\theta=0.2$ & 11.1935 & 11.1687 & 10.6107 & $z_{1}>z_{2}>z_{3}$ \\
$\theta=0.3$ & 11.1805 & 11.1617 & 10.6307 & $z_{1}>z_{2}>z_{3}$ \\
$\theta=0.4$ & 11.1666 & 11.1560 & 10.6502 & $z_{1}>z_{2}>z_{3}$ \\
$\theta=0.5$ & 11.1517 & 11.1519 & 10.6693 & $z_{2}>z_{1}>z_{3}$ \\
$\theta=0.6$ & 11.1374 & 11.1477 & 10.6878 & $z_{2}>z_{1}>z_{3}$ \\
$\theta=0.7$ & 11.1261 & 11.1387 & 10.7082 & $z_{2}>z_{1}>z_{3}$ \\
$\theta=0.8$ & 11.1394 & 11.0926 & 10.7409 & $z_{2}>z_{1}>z_{3}$ \\
$\theta=0.9$ & 11.1641 & 11.0679 & 10.7409 & $z_{2}>z_{1}>z_{3}$ \\
$\theta=1.0$ & 11.1891 & 11.0428 & 10.7409 & $z_{2}>z_{1}>z_{3}$ \\
\hline
\end{tabular}

TABLE 3: The ranking results with different $\theta$.

\begin{tabular}{lccccl}
\hline & $\varepsilon_{1}$ & $\varepsilon_{2}$ & $\varepsilon_{3}$ & $\varepsilon_{4}$ & Ranking results \\
\hline$\theta=0$ & 12.0645 & 12.7036 & 12.7192 & 12.5482 & $z_{3}>z_{2}>z_{4}>z_{1}$ \\
$\theta=0.1$ & 12.1504 & 12.6811 & 12.6274 & 12.5768 & $z_{2}>z_{3}>z_{4}>z_{1}$ \\
$\theta=0.2$ & 12.2387 & 12.6580 & 12.5329 & 12.6059 & $z_{2}>z_{4}>z_{3}>z_{1}$ \\
$\theta=0.3$ & 12.3235 & 12.6342 & 12.4818 & 12.5961 & $z_{2}>z_{4}>z_{3}>z_{1}$ \\
$\theta=0.4$ & 12.4058 & 12.6095 & 12.4348 & 12.5853 & $z_{2}>z_{4}>z_{3}>z_{1}$ \\
$\theta=0.5$ & 12.5129 & 12.5636 & 12.3874 & 12.5716 & $z_{4}>z_{2}>z_{1}>z_{3}$ \\
$\theta=0.6$ & 12.6462 & 12.4867 & 12.3483 & 12.5544 & $z_{1}>z_{4}>z_{2}>z_{3}$ \\
$\theta=0.7$ & 12.8194 & 12.3218 & 12.3736 & 12.5208 & $z_{1}>z_{4}>z_{3}>z_{2}$ \\
$\theta=0.8$ & 12.9711 & 12.1699 & 12.4113 & 12.4831 & $z_{1}>z_{4}>z_{3}>z_{2}$ \\
$\theta=0.9$ & 13.1231 & 12.0569 & 12.4122 & 12.4432 & $z_{1}>z_{4}>z_{3}>z_{2}$ \\
$\theta=1.0$ & 13.2799 & 11.9645 & 12.3986 & 12.3925 & $z_{1}>z_{3}>z_{4}>z_{2}$ \\
\hline
\end{tabular}

evaluation problem, three decision makers use the following five attributes: total investment amount $\left(c_{1}\right)$, profit ratio of investment $\left(c_{2}\right)$, internal rate of return $\left(c_{3}\right)$, loan repayment rate $\left(c_{4}\right)$, and investment recovery period $\left(c_{5}\right)$, to evaluate the investment project. The set of all alternatives is denoted by $z=\left(z_{1}, z_{2}, z_{3}, z_{4}\right)$. The set of evaluative attribute is denoted by $C=\left(c_{1}, c_{2}, c_{3}, c_{4}, c_{5}\right)$, where $C_{\mathrm{I}}=\left(c_{1}, c_{2}, c_{3}\right)$ and $C_{\mathrm{II}}=$ $\left(c_{4}, c_{5}\right)$. We use the GITFNWGA operator [32] to combine the opinions of the three decision makers to obtain an average weighting matrix and an average decision matrix. Based on the two average matrices, the importance weight $\omega_{j}$ of each $c_{j}$ and the evaluative ratings of each $z_{i}$ with respect to $c_{j}$ are shown in Table 7.

Using the proposed method for IT2TrFS MCDA, we obtain different ranking results of alternatives with different risk preference coefficients, which are presented in Table 3.

According to this table, we can conclude that when a decision maker is absolutely risk averse, namely, $\theta=0$, the best selection is program $z_{3}$; when $\theta \in[0.1,0.4]$, the best selection is program $z_{2}$; when the decision maker is 
TABLE 4: Comparison analysis of the obtained results.

\begin{tabular}{|c|c|c|}
\hline Research source & Comparative methods & Ranking results \\
\hline \multirow{3}{*}{ The supplier selection problem } & The likelihood-based MCDA method [25] & $z_{1}>z_{2}>z_{3}$ \\
\hline & The linear assignment method [33] & $z_{2}>z_{3}, z_{2}>z_{1}$ \\
\hline & The extended QUALIFLEX method [7] & $z_{2}>z_{1}>z_{3}$ \\
\hline \multirow{3}{*}{ The engineering investment project evaluation problems } & The likelihood-based MCDA method [25] & $z_{4}>z_{2}>z_{1}>z_{3}$ \\
\hline & The linear assignment method [33] & $z_{2}>z_{4}>z_{3}>z_{1}$ \\
\hline & The extended QUALIFLEX method [7] & $z_{2}>z_{4}>z_{3}>z_{1}$ \\
\hline
\end{tabular}

risk neutral, namely, $\theta=0.5$, the best selection is program $z_{4}$; when the decision maker is risk preferred, namely, $\theta \in$ $[0.6,1]$, the best selection is program $z_{1}$. According to the actual situation, the company is in its infancy and decision makers need to make risk decisions to promote the rapid growth of the company. Thus, decision makers tend toward risk preference, and the risk preference coefficient is 0.8 , which means that the final option is $z_{1}$.

6.3. Algorithm Comparison Analysis. As discussed, the supplier selection for the aviation project and the engineering investment project evaluation problem verify the validity and feasibility of the proposed method. In this section, we further illustrate the superiority of the proposed method through comparison analysis.

We have chosen several classical algorithms of the existing IT2FS MADM including an interval type-2 fuzzy PROMETHEE method using a likelihood-based outranking comparison approach [25]. A linear assignment method for multicriteria decision analysis with IT2FS [33], the extended QUALIFLEX method for multicriteria decision analysis is based on IT2FS and applications to medical decision making [7]. As shown in Table 4, in the aviation supplier selection problem, through the likelihood-based MCDA method, we can obtain the final ranking of suppliers as $z_{1}>z_{2}>z_{3}$; through the linear assignment method, we can obtain the final option as $z_{2}$, but we cannot rank $z_{1}$ and $z_{3}$; through the extended QUALIFLEX method, we can obtain the final ranking of suppliers as $z_{2}>z_{1}>z_{3}$. Given the preceding results, we can conclude that the likelihood-based MCDA method and the extended QUALIFLEX method are superior to the linear assignment method because these two programs can provide not only the best solutions but also the ranking of programs. However, neither of these two methods take into account the risk preferences; the results show only an extreme case of the proposed method. For example, the final ranking of the likelihood-based MCDA method is $z_{1}>z_{2}>z_{3}$, which is the same as the results of the method proposed in this study when decision makers are inclined to risk aversion, namely, $\theta \in[0,0.4]$. The final ranking of the extended QUALIFLEX method is $z_{2}>z_{1}>z_{3}$, which is the same as the results of our proposed method when decision makers are neutral or inclined to risk preference, namely, $\theta \in[0.5,1.0]$. In reality, according to risk appetite and enterprise development, the airline decision makers select the risk preference coefficient 0.2 and then the final option is $z_{1}$. Therefore, the proposed
TABLE 5: The corresponding weights $W_{j}$ of criterion $c_{j}$.

\begin{tabular}{ll}
\hline & Corresponding weight $W_{j}$ of attribute $c_{j}$ \\
\hline$c_{1}$ & {$[(1.00001 .00001 .00001 .0000 ; 0.8)$,} \\
& $(1.00001 .00001 .00001 .0000 ; 1.0)] ;$ \\
\hline \multirow{2}{*}{$c_{2}$} & {$[(0.00750 .00750 .01500 .0525 ; 0.8)$,} \\
& $(0.00000 .00000 .02000 .0700 ; 1.0)] ;$ \\
$c_{3}$ & {$[(0.08750 .12000 .16000 .1825 ; 0.8)$,} \\
& $(0.04000 .10000 .18000 .2300 ; 1.0)] ;$ \\
$c_{4}$ & {$[(1.00001 .00001 .00001 .0000 ; 0.8)$,} \\
& $(1.00001 .00001 .00001 .0000 ; 1.0)] ;$ \\
$c_{5}$ & {$[(0.23250 .25500 .32500 .3575 ; 0.8)$,} \\
& $(0.17000 .22000 .36000 .4200 ; 1.0)] ;$ \\
$c_{6}$ & {$[(0.40250 .45250 .53750 .5675 ; 0.8)$,} \\
& $(0.32000 .41000 .58000 .6500 ; 1.0)] ;$ \\
$c_{7}$ & {$[(0.94750 .98500 .99250 .9925 ; 0.8)$,} \\
& $(0.93000 .98001 .00001 .0000 ; 1.0)] ;$ \\
\hline \multirow{2}{*}{$c_{8}$} & {$[(0.65000 .67250 .75750 .7900 ; 0.8)$,} \\
& $(0.58000 .63000 .80000 .8600 ; 1.0)] ;$ \\
$c_{9}$ & {$[(0.78250 .81500 .88500 .9075 ; 0.8)$,} \\
& $(0.72000 .78000 .92000 .9700 ; 1.0)]$ \\
\hline
\end{tabular}

method not only can obtain a complete sort of result, but also can fully reflect the flexibility of the decision process and the subjective views of decision makers.

For the engineering investment project evaluation problem, through the likelihood-based MCDA method, we can obtain the final ranking of the program as $z_{4}>z_{2}>z_{1}>z_{3}$; through the linear assignment method, we can obtain the final ranking of the program as $z_{2}>z_{4}>z_{3}>z_{1}$; through the extended QUALIFLEX method, we can obtain the final ranking of the program as $z_{2}>z_{4}>z_{3}>z_{1}$. As the preceding findings show, all of the ranking results of the three methods are an extreme case of this proposed method. For example, the final ranking of the likelihood-based MCDA method is $z_{4}>z_{2}>z_{1}>z_{3}$, which is the same as the result of our proposed method when $\theta=0.5$; the final ranking of the linear assignment method is $z_{2}>z_{4}>z_{3}>z_{1}$, which is the same as the result of our proposed method when $\theta \in[0.2,0.4]$; the final ranking of the extended QUALIFLEX method is $z_{2}>$ $z_{4}>z_{3}>z_{1}$, which is the same as the result of our proposed method when $\theta \in[0.2,0.4]$. According to the actual situation, the company is at the initial stage and needs bold decisions to facilitate its rapid growth, so the decision makers choose risk 
TABLE 6: The evaluative rating of the alternative $z_{i}$ with respect to the criterion $c_{j}$.

\begin{tabular}{|c|c|}
\hline \multicolumn{2}{|c|}{ Evaluation value of alternative $z_{1}$ with respect to $c_{j} \in C$} \\
\hline$c_{1}$ & [(0.9475 0.9850 0.9925 0.9925; 0.8), (0.8300 0.8800 1.0000 1.0000; 1.0)]; \\
\hline$c_{2}$ & [(0.6500 $0.67250 .75750 .7900 ; 0.8),(0.58000 .63000 .80000 .8600 ; 1.0)]$ \\
\hline$c_{3}$ & 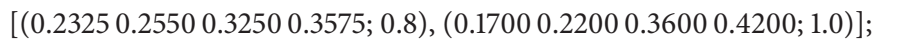 \\
\hline$c_{4}$ & [(0.9475 0.9850 $0.99250 .9925 ; 0.8),(0.83000 .88001 .00001 .0000 ; 1.0)]$ \\
\hline$c_{5}$ & [(0.0875 0.1200 $0.16000 .1825 ; 0.8),(0.04000 .10000 .18000 .2300 ; 1.0)]$ \\
\hline$c_{6}$ & [(0.0875 $0.12000 .16000 .1825 ; 0.8),(0.04000 .10000 .18000 .2300 ; 1.0)]$ \\
\hline$c_{7}$ & [(0.4025 $0.45250 .53750 .5675 ; 0.8),(0.32000 .41000 .58000 .6500 ; 1.0)]$ \\
\hline$c_{8}$ & [(0.7825 $0.81500 .88500 .9075 ; 0.8),(0.72000 .78000 .92000 .9700 ; 1.0)]$ \\
\hline$c_{9}$ & 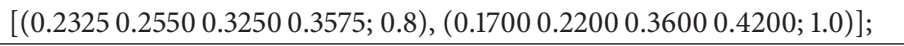 \\
\hline \multicolumn{2}{|c|}{ Evaluation value of alternative $z_{2}$ with respect to $c_{j} \in C$} \\
\hline$c_{1}$ & [(0.9475 $0.98500 .99250 .9925 ; 0.8),(0.83000 .88001 .00001 .0000 ; 1.0)]$ \\
\hline$c_{2}$ & [(0.7825 $0.81500 .88500 .9075 ; 0.8),(0.72000 .78000 .92000 .9700 ; 1.0)] ;$ \\
\hline$c_{3}$ & [(0.0875 $0.12000 .16000 .1825 ; 0.8),(0.04000 .10000 .18000 .2300 ; 1.0)]$ \\
\hline$c_{4}$ & [(0.0075 $0.00750 .01500 .0525 ; 0.8),(0.00000 .00000 .02000 .0700 ; 1.0)]$ \\
\hline$c_{5}$ & [(0.7825 0.8150 0.8850 0.9075; 0.8), (0.7200 $0.78000 .92000 .9700 ; 1.0)]$ \\
\hline$c_{6}$ & [(0.0875 $0.12000 .16000 .1825 ; 0.8),(0.04000 .10000 .18000 .2300 ; 1.0)]$ \\
\hline$c_{7}$ & 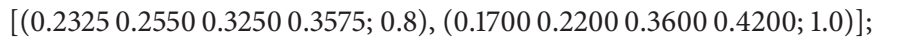 \\
\hline$c_{8}$ & [(1.0000 1.0000 1.0000 1.0000; 0.8), (1.0000 1.0000 1.0000 1.0000; 1.0)]; \\
\hline$c_{9}$ & [(0.9475 $0.98500 .99250 .9925 ; 0.8),(0.83000 .88001 .00001 .0000 ; 1.0)]$ \\
\hline \multicolumn{2}{|c|}{ Evaluation value of alternative $z_{3}$ with respect to $c_{j} \in C$} \\
\hline$c_{1}$ & [(0.4025 0.4525 $0.53750 .5675 ; 0.8),(0.32000 .41000 .58000 .6500 ; 1.0)]$ \\
\hline$c_{2}$ & [(0.0875 $0.12000 .16000 .1825 ; 0.8),(0.04000 .10000 .18000 .2300 ; 1.0)] ;$ \\
\hline$c_{3}$ & [(0.0000 0.0000 $0.00000 .0000 ; 0.8),(0.00000 .00000 .00000 .0000 ; 1.0)]$; \\
\hline$c_{4}$ & [(0.7825 $0.81500 .88500 .9075 ; 0.8),(0.72000 .78000 .92000 .9700 ; 1.0)]$ \\
\hline$c_{5}$ & [(0.9475 $0.98500 .99250 .9925 ; 0.8),(0.83000 .88001 .00001 .0000 ; 1.0)]$ \\
\hline$c_{6}$ & [(0.6500 0.6725 $0.75750 .7900 ; 0.8),(0.58000 .63000 .80000 .8600 ; 1.0)]$ \\
\hline$c_{7}$ & [(0.4025 0.4525 0.5375 0.5675; 0.8), (0.3200 $0.41000 .58000 .6500 ; 1.0)]$ \\
\hline$c_{8}$ & [(0.9475 $0.98500 .99250 .9925 ; 0.8),(0.83000 .88001 .00001 .0000 ; 1.0)]$ \\
\hline$c_{9}$ & [(0.7825 $0.81500 .88500 .9075 ; 0.8),(0.72000 .78000 .92000 .9700 ; 1.0)]$ \\
\hline
\end{tabular}

preference coefficient 0.8 and the final choice of investment program is $z_{1}$. This example further confirms the advantages of the proposed method. When the comparison methods are used, one can go against the wishes of policymakers and make an inappropriate choice of programs.

Of course, because of the different research questions and initial program data, not every interval type-2 fuzzy decision problem presents the results of these two examples. Sometimes, different risk preference coefficients can obtain results similar to the decision-making results; sometimes, from risk aversion to risk neutrality and risk preference, one change is observed in the ranking results (Section 6.1); sometimes, with the change of $\theta$, the ranking results also change many times (Section 6.2). However, overall, with the change of $\theta$, different results have existing larger likelihood, so decision makers need to select the appropriate results based on actual risk preference.

The given two examples can fully reflect the advantages of the proposed method.

First, the proposed algorithm fully reflects the flexibility of the decision-making process, which means that decision makers can select different $\theta$ to obtain different decision results according to their own risk preference and the actual decision environment. Compared with current methods, the proposed algorithm is more reasonable.

Second, the proposed method can be used widely, which means that, for the actual decision problems, regardless of the risk preference of decision makers and the decision environment, all decision makers can obtain reasonable decision results based on the risk preference coefficient. However, the current methods can obtain decision results based on only one situation.

Finally, the proposed method is easy to implement on a computer and has a good potential to solve practical problems. At present, the method has been successfully applied to practical engineering problems with different risk preferences, such as supplier selection in aviation projects and engineering investment project evaluation.

\section{Conclusion}

IT2TFS have more advantages in describing uncertain information than the general fuzzy sets. IT2TrF can effectively describe qualitative indices [7, 24, 25, 33]. In this study, 
TABLE 7: The collective IT2TrF data using the GITFNWGA operator in the engineering investment project evaluation problem.

\begin{tabular}{|c|c|}
\hline \multicolumn{2}{|r|}{ The importance weights of each $\omega_{j}$ of each $c_{j}$} \\
\hline$C_{1}$ & [(0.90 0.97 0.97 0.98; 0.8), (0.83 0.97 0.97 1.00; 1.0)]; \\
\hline$C_{2}$ & [(0.90 0.97 0.97 0.98; 0.8), (0.83 0.97 0.97 1.00; 1.0)]; \\
\hline$C_{3}$ & [(0.53 $0.630 .630 .73 ; 0.8),(0.430 .630 .630 .83 ; 1.0)]$ \\
\hline$C_{4}$ & [(0.85 0.93 0.93 0.97; 0.8), (0.77 0.93 0.93 1.00; 1.0)]; \\
\hline$C_{5}$ & {$[(0.680 .700 .750 .80 ; 0.8),(0.560 .700 .780 .90 ; 1.0)] ;$} \\
\hline \multicolumn{2}{|r|}{ The evaluative ratings of each $z_{i}$ with respect to $c_{j}$} \\
\hline \multicolumn{2}{|l|}{$z_{1}$} \\
\hline$C_{1}$ & [( $0.730 .780 .900 .94 ; 0.8),(0.730 .780 .900 .94 ; 1.0)] ;$ \\
\hline$C_{2}$ & {$[(0.560 .630 .770 .82 ; 0.8),(0.560 .630 .770 .82 ; 1.0)] ;$} \\
\hline$C_{3}$ & [(0.78 $0.840 .950 .98 ; 0.8),(0.780 .840 .950 .98 ; 1.0)] ;$ \\
\hline$C_{4}$ & [(0.88 0.94 0.99 0.99; 0.8), (0.85 0.91 0.97 1.00; 1.0)]; \\
\hline$C_{5}$ & [( $0.420 .500 .600 .75 ; 0.8),\left(\begin{array}{lllll}0 & 0.40 & 0.48 & 0.50 & 0.75 ; 1.0\end{array}\right)$; \\
\hline \multicolumn{2}{|l|}{$z_{2}$} \\
\hline$C_{1}$ & [(0.67 0.73 $0.880 .93 ; 0.8),(0.670 .730 .880 .93 ; 1.0)] ;$ \\
\hline$C_{2}$ & {$[(0.550 .750 .930 .95 ; 0.8),(0.760 .800 .930 .95 ; 1.0)] ;$} \\
\hline$C_{3}$ & [(0.85 0.91 0.97 0.99; 0.8), (0.85 0.91 $0.970 .99 ; 1.0)]$ \\
\hline $\mathrm{C}_{4}$ & [(0.79 0.85 0.93 0.95; 0.8), (0.79 0.85 $0.930 .95 ; 1.0)]$ \\
\hline$C_{5}$ & [( $0.320 .480 .840 .90 ; 0.8),(0.220 .600 .840 .90 ; 1.0)] ;$ \\
\hline \multicolumn{2}{|l|}{$z_{3}$} \\
\hline$C_{1}$ & [(0.85 0.91 0.97 0.99; 0.8), (0.85 0.91 $0.970 .99 ; 1.0)]$ \\
\hline $\mathrm{C}_{2}$ & {$[(0.560 .630 .770 .82 ; 0.8),(0.560 .650 .770 .82 ; 1.0)]$} \\
\hline$C_{3}$ & [(0.42 $0.550 .700 .80 ; 0.8),(0.530 .600 .780 .90 ; 1.0)] ;$ \\
\hline $\mathrm{C}_{4}$ & [(0.79 $0.850 .930 .95 ; 0.8),(0.790 .850 .930 .95 ; 1.0)] ;$ \\
\hline$C_{5}$ & {$[(0.770 .820 .881 .00 ; 0.8),(0.840 .960 .981 .0 ; 1.0)]$} \\
\hline \multicolumn{2}{|l|}{$z_{4}$} \\
\hline$C_{1}$ & [(0.73 0.78 0.90 0.94; 0.8), (0.73 $0.780 .900 .94 ; 1.0)]$ \\
\hline$C_{2}$ & {$[(0.460 .550 .700 .75 ; 0.8),(0.460 .550 .700 .75 ; 1.0)] ;$} \\
\hline$C_{3}$ & [( $\left.0.700 .730 .880 .98 ; 0.8),\left(\begin{array}{llllll}0.67 & 0.73 & 0.88 & 0.94 ; 1.0\end{array}\right)\right]$ \\
\hline$C_{4}$ & [(0.85 0.91 0.97 0.99; 0.8), (0.85 0.91 0.97 0.99; 1.0)]; \\
\hline$C_{5}$ & {$[(0.930 .981 .001 .00 ; 0.8),(0.930 .981 .001 .00 ; 1.0)] ;$} \\
\hline
\end{tabular}

considering the background of the MADM problem, we conclude the following three research tasks.
(1) We propose the measurement method of risk preference for IT2TrF decision makers. Based on different attitudes of decision makers that have different risk preference toward the uncertainty range of IT2TrF, the method measures the risk preference coefficient of decision makers. This method applies to both IT2TFS and IT2FS.

(2) We propose the calculation method of preference likelihood of trapezoidal fuzzy numbers. This method is simple to calculate and easy to implement on a computer. Using the formula of likelihood, we establish the ranking method of preference relations of attributes, which applies not only to decision problems that have given attribute weights and IT2TrF but also to decision problems that have given attribute weights and exact value.

(3) From the subjective point of view, considering the different risk preferences of decision makers, we introduce the risk preference coefficient and propose a likelihood decision-making algorithm considering the risk preferences of decision makers. Through example analysis and algorithm comparison, the algorithm is proven reasonable.

In this study, the proposed algorithm is mainly applied to IT2TrF. However, the basic idea of this algorithm is also applicable to all IT2TFS. The proposed algorithm can be further extended to the interval Gaussian type-2 fuzzy set [34] and further examine the IT2TFS multiattribute group decision-making problems considering the risk preferences of decision makers.

\section{Appendices}

\section{A. The Initial Data of Application Research}

The initial data of application research are established in Tables 5, 6, and 7 .

\section{B. Demonstration of the Properties of Likelihood}

Appendix B provides a demonstration of the properties of likelihood $L\left(A_{\rho} \geq A_{\beta}\right)$.

Property B.1. If at least one of $h_{A \rho} \neq h_{A \beta}, a_{1 \rho} \neq a_{4 \rho}, a_{1 \beta} \neq a_{4 \beta}$, and $a_{\xi \beta} \neq a_{\xi \beta}(\xi=1,2,3,4)$ holds, we can obtain

$$
\begin{aligned}
& 0 \leq \max \left[\frac{\sum_{\xi=1}^{4} \max \left(a_{\xi \beta}-a_{\xi \rho}, 0\right)+\left(a_{4 \beta}-a_{1 \rho}\right)+2 \max \left(h_{A \beta}-h_{A \rho}, 0\right)}{\sum_{\xi=1}^{4}\left|a_{\xi \beta}-a_{\xi \rho}\right|+\left(a_{4 \rho}-a_{1 \rho}\right)+\left(a_{4 \beta}-a_{1 \beta}\right)+2\left|h_{A \beta}-h_{A \rho}\right|}, 0\right]<\infty \\
& \Longrightarrow-\infty \leq 1-\max \left[\frac{\sum_{\xi=1}^{4} \max \left(a_{\xi \beta}-a_{\xi \rho}, 0\right)+\left(a_{4 \beta}-a_{1 \rho}\right)+2 \max \left(h_{A \beta}-h_{A \rho}, 0\right)}{\sum_{\xi=1}^{4}\left|a_{\xi \beta}-a_{\xi \rho}\right|+\left(a_{4 \rho}-a_{1 \rho}\right)+\left(a_{4 \beta}-a_{1 \beta}\right)+2\left|h_{A \beta}-h_{A \rho}\right|}, 0\right]<0 \\
& \Longrightarrow 0 \leq \max \left\{1-\max \left[\frac{\sum_{\xi=1}^{4} \max \left(a_{\xi \beta}-a_{\xi \rho}, 0\right)+\left(a_{4 \beta}-a_{1 \rho}\right)+2 \max \left(h_{A \beta}-h_{A \rho}, 0\right)}{\sum_{\xi=1}^{4}\left|a_{\xi \beta}-a_{\xi \rho}\right|+\left(a_{4 \rho}-a_{1 \rho}\right)+\left(a_{4 \beta}-a_{1 \beta}\right)+2\left|h_{A \beta}-h_{A \rho}\right|}, 0\right], 0\right\} \leq 1 .
\end{aligned}
$$

Namely, $0 \leq L\left(A_{\rho} \geq A_{\beta}\right) \leq 1$. 
Property B.2. Given

$$
\begin{aligned}
& L\left(A_{\rho} \geq A_{\beta}\right)=\max \left\{1-\max \left[\frac{\sum_{\xi=1}^{4} \max \left(a_{\xi \beta}-a_{\xi \rho}, 0\right)+\left(a_{4 \beta}-a_{1 \rho}\right)+2 \max \left(h_{A \beta}-h_{A \rho}, 0\right)}{\sum_{\xi=1}^{4}\left|a_{\xi \beta}-a_{\xi \rho}\right|+\left(a_{4 \rho}-a_{1 \rho}\right)+\left(a_{4 \beta}-a_{1 \beta}\right)+2\left|h_{A \beta}-h_{A \rho}\right|}, 0\right], 0\right\} \\
& L\left(A_{\beta} \geq A_{\rho}\right)=\max \left\{1-\max \left[\frac{\sum_{\xi=1}^{4} \max \left(a_{\xi \rho}-a_{\xi \beta}, 0\right)+\left(a_{4 \rho}-a_{1 \beta}\right)+2 \max \left(h_{A \rho}-h_{A \beta}, 0\right)}{\sum_{\xi=1}^{4}\left|a_{\xi \rho}-a_{\xi \beta}\right|+\left(a_{4 \rho}-a_{1 \rho}\right)+\left(a_{4 \beta}-a_{1 \beta}\right)+2\left|h_{A \rho}-h_{A \beta}\right|}, 0\right], 0\right\} \\
& \Longrightarrow \sum_{\xi=1}^{4} \max \left(a_{\xi \beta}-a_{\xi \rho}, 0\right)+\sum_{\xi=1}^{4} \max \left(a_{\xi \rho}-a_{\xi \beta}, 0\right)=\sum_{\xi=1}^{4}\left|a_{\xi \rho}-a_{\xi \beta}\right| \\
& 2 \max \left(h_{A \beta}-h_{A \rho}, 0\right)+2 \max \left(h_{A \rho}-h_{A \beta}, 0\right)=2\left|h_{A \rho}-h_{A \beta}\right| \\
& \frac{\sum_{\xi=1}^{4} \max \left(a_{\xi \beta}-a_{\xi \rho}, 0\right)+\left(a_{4 \beta}-a_{1 \rho}\right)+2 \max \left(h_{A \beta}-h_{A \rho}, 0\right)}{\sum_{\xi=1}^{4}\left|a_{\xi \beta}-a_{\xi \rho}\right|+\left(a_{4 \rho}-a_{1 \rho}\right)+\left(a_{4 \beta}-a_{1 \beta}\right)+2\left|h_{A \beta}-h_{A \rho}\right|} \\
& +\frac{\sum_{\xi=1}^{4} \max \left(a_{\xi \rho}-a_{\xi \beta}, 0\right)+\left(a_{4 \rho}-a_{1 \beta}\right)+2 \max \left(h_{A \rho}-h_{A \beta}, 0\right)}{\sum_{\xi=1}^{4}\left|a_{\xi \rho}-a_{\xi \beta}\right|+\left(a_{4 \rho}-a_{1 \rho}\right)+\left(a_{4 \beta}-a_{1 \beta}\right)+2\left|h_{A \rho}-h_{A \beta}\right|}=1 \\
& \Longrightarrow L\left(A_{\rho} \geq A_{\beta}\right)=\max \left\{1-\max \left[\frac{\sum_{\xi=1}^{4} \max \left(a_{\xi \beta}-a_{\xi \rho}, 0\right)+\left(a_{4 \beta}-a_{1 \rho}\right)+2 \max \left(h_{A \beta}-h_{A \rho}, 0\right)}{\sum_{\xi=1}^{4}\left|a_{\xi \beta}-a_{\xi \rho}\right|+\left(a_{4 \rho}-a_{1 \rho}\right)+\left(a_{4 \beta}-a_{1 \beta}\right)+2\left|h_{A \beta}-h_{A \rho}\right|}, 0\right], 0\right\} \\
& =\max \left\{1-\max \left[1-\frac{\sum_{\xi=1}^{4} \max \left(a_{\xi \rho}-a_{\xi \beta}, 0\right)+\left(a_{4 \rho}-a_{1 \beta}\right)+2 \max \left(h_{A \rho}-h_{A \beta}, 0\right)}{\sum_{\xi=1}^{4}\left|a_{\xi \rho}-a_{\xi \beta}\right|+\left(a_{4 \rho}-a_{1 \rho}\right)+\left(a_{4 \beta}-a_{1 \beta}\right)+2\left|h_{A \rho}-h_{A \beta}\right|}, 0\right], 0\right\} \\
& =\max \left\{\min \left[1-\frac{\sum_{\xi=1}^{4} \max \left(a_{\xi \rho}-a_{\xi \beta}, 0\right)+\left(a_{4 \rho}-a_{1 \beta}\right)+2 \max \left(h_{A \rho}-h_{A \beta}, 0\right)}{\sum_{\xi=1}^{4}\left|a_{\xi \rho}-a_{\xi \beta}\right|+\left(a_{4 \rho}-a_{1 \rho}\right)+\left(a_{4 \beta}-a_{1 \beta}\right)+2\left|h_{A \rho}-h_{A \beta}\right|}, 1\right], 0\right\} \\
& L\left(A_{\beta} \geq A_{\rho}\right)=\max \left\{1-\max \left[\frac{\sum_{\xi=1}^{4} \max \left(a_{\xi \rho}-a_{\xi \beta}, 0\right)+\left(a_{4 \rho}-a_{1 \beta}\right)+2 \max \left(h_{A \rho}-h_{A \beta}, 0\right)}{\sum_{\xi=1}^{4}\left|a_{\xi \rho}-a_{\xi \beta}\right|+\left(a_{4 \rho}-a_{1 \rho}\right)+\left(a_{4 \beta}-a_{1 \beta}\right)+2\left|h_{A \rho}-h_{A \beta}\right|}, 0\right], 0\right\} \\
& =\max \left\{\min \left[\frac{\sum_{\xi=1}^{4} \max \left(a_{\xi \rho}-a_{\xi \beta}, 0\right)+\left(a_{4 \rho}-a_{1 \beta}\right)+2 \max \left(h_{A \rho}-h_{A \beta}, 0\right)}{\sum_{\xi=1}^{4}\left|a_{\xi \rho}-a_{\xi \beta}\right|+\left(a_{4 \rho}-a_{1 \rho}\right)+\left(a_{4 \beta}-a_{1 \beta}\right)+2\left|h_{A \rho}-h_{A \beta}\right|}, 1\right], 0\right\},
\end{aligned}
$$

$$
\begin{aligned}
& \min \left[1-\frac{\sum_{\xi=1}^{4} \max \left(a_{\xi \rho}-a_{\xi \beta}, 0\right)+\left(a_{4 \rho}-a_{1 \beta}\right)+2 \max \left(h_{A \rho}-h_{A \beta}, 0\right)}{\sum_{\xi=1}^{4}\left|a_{\xi \rho}-a_{\xi \beta}\right|+\left(a_{4 \rho}-a_{1 \rho}\right)+\left(a_{4 \beta}-a_{1 \beta}\right)+2\left|h_{A \rho}-h_{A \beta}\right|}, 1\right] \leq 0 \\
& \Longrightarrow \frac{\sum_{\xi=1}^{4} \max \left(a_{\xi \rho}-a_{\xi \beta}, 0\right)+\left(a_{4 \rho}-a_{1 \beta}\right)+2 \max \left(h_{A \rho}-h_{A \beta}, 0\right)}{\sum_{\xi=1}^{4}\left|a_{\xi \rho}-a_{\xi \beta}\right|+\left(a_{4 \rho}-a_{1 \rho}\right)+\left(a_{4 \beta}-a_{1 \beta}\right)+2\left|h_{A \rho}-h_{A \beta}\right|} \geq 1 \\
& \Longrightarrow L\left(A_{\rho} \geq A_{\beta}\right)+L\left(A_{\beta} \geq A_{\rho}\right) \\
& =\max \left\{\min \left[1-\frac{\sum_{\xi=1}^{4} \max \left(a_{\xi \rho}-a_{\xi \beta}, 0\right)+\left(a_{4 \rho}-a_{1 \beta}\right)+2 \max \left(h_{A \rho}-h_{A \beta}, 0\right)}{\sum_{\xi=1}^{4}\left|a_{\xi \rho}-a_{\xi \beta}\right|+\left(a_{4 \rho}-a_{1 \rho}\right)+\left(a_{4 \beta}-a_{1 \beta}\right)+2\left|h_{A \rho}-h_{A \beta}\right|}, 1\right], 0\right\} \\
& \quad+\max \left\{\min \left[\frac{\sum_{\xi=1}^{4} \max \left(a_{\xi \rho}-a_{\xi \beta}, 0\right)+\left(a_{4 \rho}-a_{1 \beta}\right)+2 \max \left(h_{A \rho}-h_{A \beta}, 0\right)}{\sum_{\xi=1}^{4}\left|a_{\xi \rho}-a_{\xi \beta}\right|+\left(a_{4 \rho}-a_{1 \rho}\right)+\left(a_{4 \beta}-a_{1 \beta}\right)+2\left|h_{A \rho}-h_{A \beta}\right|}, 1\right], 0\right\}=0+\max (1,0)=1
\end{aligned}
$$


(2) if

$$
\begin{aligned}
& \min \left[1-\frac{\sum_{\xi=1}^{4} \max \left(a_{\xi \rho}-a_{\xi \beta}, 0\right)+\left(a_{4 \rho}-a_{1 \beta}\right)+2 \max \left(h_{A \rho}-h_{A \beta}, 0\right)}{\sum_{\xi=1}^{4}\left|a_{\xi \rho}-a_{\xi \beta}\right|+\left(a_{4 \rho}-a_{1 \rho}\right)+\left(a_{4 \beta}-a_{1 \beta}\right)+2\left|h_{A \rho}-h_{A \beta}\right|}, 1\right]>0 \\
& \Longrightarrow 0 \leq \frac{\sum_{\xi=1}^{4} \max \left(a_{\xi \rho}-a_{\xi \beta}, 0\right)+\left(a_{4 \rho}-a_{1 \beta}\right)+2 \max \left(h_{A \rho}-h_{A \beta}, 0\right)}{\sum_{\xi=1}^{4}\left|a_{\xi \rho}-a_{\xi \beta}\right|+\left(a_{4 \rho}-a_{1 \rho}\right)+\left(a_{4 \beta}-a_{1 \beta}\right)+2\left|h_{A \rho}-h_{A \beta}\right|}<1 \\
& \Longrightarrow L\left(A_{\rho} \geq A_{\beta}\right)+L\left(A_{\beta} \geq A_{\rho}\right) \\
& =\max \left\{\min \left[1-\frac{\sum_{\xi=1}^{4} \max \left(a_{\xi \rho}-a_{\xi \beta}, 0\right)+\left(a_{4 \rho}-a_{1 \beta}\right)+2 \max \left(h_{A \rho}-h_{A \beta}, 0\right)}{\sum_{\xi=1}^{4}\left|a_{\xi \rho}-a_{\xi \beta}\right|+\left(a_{4 \rho}-a_{1 \rho}\right)+\left(a_{4 \beta}-a_{1 \beta}\right)+2\left|h_{A \rho}-h_{A \beta}\right|}, 1\right], 0\right\} \\
& +\max \left\{\min \left[\frac{\sum_{\xi=1}^{4} \max \left(a_{\xi \rho}-a_{\xi \beta}, 0\right)+\left(a_{4 \rho}-a_{1 \beta}\right)+2 \max \left(h_{A \rho}-h_{A \beta}, 0\right)}{\sum_{\xi=1}^{4}\left|a_{\xi \rho}-a_{\xi \beta}\right|+\left(a_{4 \rho}-a_{1 \rho}\right)+\left(a_{4 \beta}-a_{1 \beta}\right)+2\left|h_{A \rho}-h_{A \beta}\right|}, 1\right], 0\right\} \\
& =\min \left[1-\frac{\sum_{\xi=1}^{4} \max \left(a_{\xi \rho}-a_{\xi \beta}, 0\right)+\left(a_{4 \rho}-a_{1 \beta}\right)+2 \max \left(h_{A \rho}-h_{A \beta}, 0\right)}{\sum_{\xi=1}^{4}\left|a_{\xi \rho}-a_{\xi \beta}\right|+\left(a_{4 \rho}-a_{1 \rho}\right)+\left(a_{4 \beta}-a_{1 \beta}\right)+2\left|h_{A \rho}-h_{A \beta}\right|}, 1\right] \\
& +\max \left[\frac{\sum_{\xi=1}^{4} \max \left(a_{\xi \rho}-a_{\xi \beta}, 0\right)+\left(a_{4 \rho}-a_{1 \beta}\right)+2 \max \left(h_{A \rho}-h_{A \beta}, 0\right)}{\sum_{\xi=1}^{4}\left|a_{\xi \rho}-a_{\xi \beta}\right|+\left(a_{4 \rho}-a_{1 \rho}\right)+\left(a_{4 \beta}-a_{1 \beta}\right)+2\left|h_{A \rho}-h_{A \beta}\right|}, 0\right],
\end{aligned}
$$

when

$$
\begin{aligned}
0 \leq & \frac{\sum_{\xi=1}^{4} \max \left(a_{\xi \rho}-a_{\xi \beta}, 0\right)+\left(a_{4 \rho}-a_{1 \beta}\right)+2 \max \left(h_{A \rho}-h_{A \beta}, 0\right)}{\sum_{\xi=1}^{4}\left|a_{\xi \rho}-a_{\xi \beta}\right|+\left(a_{4 \rho}-a_{1 \rho}\right)+\left(a_{4 \beta}-a_{1 \beta}\right)+2\left|h_{A \rho}-h_{A \beta}\right|}<1 \\
\Longrightarrow & L\left(A_{\rho} \geq A_{\beta}\right)+L\left(A_{\beta} \geq A_{\rho}\right) \\
= & 1-\frac{\sum_{\xi=1}^{4} \max \left(a_{\xi \rho}-a_{\xi \beta}, 0\right)+\left(a_{4 \rho}-a_{1 \beta}\right)+2 \max \left(h_{A \rho}-h_{A \beta}, 0\right)}{\sum_{\xi=1}^{4}\left|a_{\xi \rho}-a_{\xi \beta}\right|+\left(a_{4 \rho}-a_{1 \rho}\right)+\left(a_{4 \beta}-a_{1 \beta}\right)+2\left|h_{A \rho}-h_{A \beta}\right|} \\
& +\left[\frac{\sum_{\xi=1}^{4} \max \left(a_{\xi \rho}-a_{\xi \beta}, 0\right)+\left(a_{4 \rho}-a_{1 \beta}\right)+2 \max \left(h_{A \rho}-h_{A \beta}, 0\right)}{\sum_{\xi=1}^{4}\left|a_{\xi \rho}-a_{\xi \beta}\right|+\left(a_{4 \rho}-a_{1 \rho}\right)+\left(a_{4 \beta}-a_{1 \beta}\right)+2\left|h_{A \rho}-h_{A \beta}\right|}, 1\right]=1,
\end{aligned}
$$

(3) if

$$
\begin{aligned}
& \frac{\sum_{\xi=1}^{4} \max \left(a_{\xi \rho}-a_{\xi \beta}, 0\right)+\left(a_{4 \rho}-a_{1 \beta}\right)+2 \max \left(h_{A \rho}-h_{A \beta}, 0\right)}{\sum_{\xi=1}^{4}\left|a_{\xi \rho}-a_{\xi \beta}\right|+\left(a_{4 \rho}-a_{1 \rho}\right)+\left(a_{4 \beta}-a_{1 \beta}\right)+2\left|h_{A \rho}-h_{A \beta}\right|} \\
& \quad<0 \\
& \quad \Longrightarrow L\left(A_{\rho} \geq A_{\beta}\right)+L\left(A_{\beta} \geq A_{\rho}\right)=0+1=1, \\
& L\left(A_{\rho} \geq A_{\beta}\right)+L\left(A_{\beta} \geq A_{\rho}\right)=1 .
\end{aligned}
$$

Property B.3. According to Property B.2, $L\left(A_{\rho} \geq A_{\beta}\right)+$ $L\left(A_{\beta} \geq A_{\rho}\right)=1$; when $L\left(A_{\rho} \geq A_{\beta}\right)=L\left(A_{\beta} \geq A_{\rho}\right)$, $L\left(A_{\rho} \geq A_{\beta}\right)=L\left(A_{\beta} \geq A_{\rho}\right)=0.5$ holds.

\section{Conflict of Interests}

The authors declare that there is no conflict of interests regarding the publication of this paper.

\section{Acknowledgments}

The work presented in this paper is supported by the National Natural Science Foundation of China (71473032, 71401027). The authors also gratefully acknowledge the helpful comments and suggestions of the reviewers and the editor Dan Simon, which have greatly improved the presentation. 


\section{References}

[1] L. A. Zadeh, "Fuzzy sets," Information and Computation, vol. 8, no. 3, pp. 338-353, 1965.

[2] L. A. Zadeh, "A theory of approximate reasoning," Machine Intelligence, vol. 9, pp. 149-194, 1979.

[3] Z. Takac, "Inclusion and subsethood measure for intervalvalued fuzzy sets and for continuous type-2 fuzzy sets," Fuzzy Sets and Systems, vol. 224, pp. 106-120, 2013.

[4] C.-T. Chen, "Extensions of the TOPSIS for group decisionmaking under fuzzy environment," Fuzzy Sets and Systems, vol. 114 , no. 1, pp. 1-9, 2000.

[5] O. Castillo, P. Melin, and W. Pedrycz, "Design of interval type-2 fuzzy models through optimal granularity allocation," Applied Soft Computing Journal, vol. 11, no. 8, pp. 5590-5601, 2011.

[6] O. Castillo and P. Melin, "Optimization of type-2 fuzzy systems based on bio-inspired methods: a concise review," Information Sciences, vol. 205, pp. 1-19, 2012.

[7] T.-Y. Chen, C.-H. Chang, and J.-F. R. Lu, "The extended QUALIFLEX method for multiple criteria decision analysis based on interval type-2 fuzzy sets and applications to medical decision making," European Journal of Operational Research, vol. 226, no. 3, pp. 615-625, 2013.

[8] D. Kahneman and A. Tversky, "Prospect theory: an analysis of decision under risk," Econometrica, vol. 47, no. 2, pp. 263-291, 1979.

[9] T.-Y. Chen, "An integrated approach for assessing criterion importance with interval type-2 fuzzy sets and signed distances," Journal of the Chinese Institute of Industrial Engineers, vol. 28, no. 8, pp. 553-572, 2011.

[10] G. Zheng, J. Xiao, Q. Jiang, and Y. Zhang, "Similarity and inclusion measures between IT2 FSs," Control and Decision, vol. 26, no. 6, pp. 861-866, 2011.

[11] M. Zarinbal, M. H. Fazel Zarandi, and I. B. Turksen, "Interval type-2 relative entropy fuzzy C-means clustering," Information Sciences, vol. 272, pp. 49-72, 2014.

[12] C.-M. Hwang, M.-S. Yang, W.-L. Hung, and E. S. Lee, "Similarity, inclusion and entropy measures between type-2 fuzzy sets based on the Sugeno integral," Mathematical and Computer Modelling, vol. 53, no. 9-10, pp. 1788-1797, 2011.

[13] C. Li, G. Zhang, J. Yi, and M. Wang, "Uncertainty degree and modeling of interval type-2 fuzzy sets: definition, method and application," Computers and Mathematics with Applications, vol. 66, no. 10, pp. 1822-1835, 2013.

[14] D. Zhai and J. M. Mendel, "Uncertainty measures for general type-2 fuzzy sets," Information Sciences, vol. 181, no. 3, pp. 503518, 2011.

[15] S.-M. Chen and L.-W. Lee, "Fuzzy multiple criteria hierarchical group decision-making based on interval type-2 fuzzy sets," IEEE Transactions on Systems, Man, and Cybernetics Part A:Systems and Humans, vol. 40, no. 5, pp. 1120-1128, 2010.

[16] S.-C. Ngan, "A type-2 linguistic set theory and its application to multi-criteria decision making," Computers and Industrial Engineering, vol. 64, no. 2, pp. 721-730, 2013.

[17] S.-M. Chen, M.-W. Yang, L.-W. Lee, and S.-W. Yang, "Fuzzy multiple attributes group decision-making based on ranking interval type-2 fuzzy sets," Expert Systems with Applications, vol. 39, no. 5, pp. 5295-5308, 2012.

[18] S. Safarzadegan Gilan, M. H. Sebt, and V. Shahhosseini, "Computing with words for hierarchical competency based selection of personnel in construction companies," Applied Soft Computing Journal, vol. 12, no. 2, pp. 860-871, 2012.
[19] S.-M. Chen and J.-H. Chen, "Fuzzy risk analysis based on similarity measures between interval-valued fuzzy numbers and interval-valued fuzzy number arithmetic operators," Expert Systems with Applications, vol. 36, no. 3, pp. 6309-6317, 2009.

[20] S.-H. Wei and S.-M. Chen, "Fuzzy risk analysis based on interval-valued fuzzy numbers," Expert Systems with Applications, vol. 36, no. 2, pp. 2285-2299, 2009.

[21] M. N. Mokhtarian, S. Sadi-nezhad, and A. Makui, "A new flexible and reliable interval valued fuzzy VIKOR method based on uncertainty risk reduction in decision making process: An application for determining a suitable location for digging some pits for municipal wet waste landfill," Computers \& Industrial Engineering, vol. 78, pp. 213-233, 2014.

[22] B. Ashtiani, F. Haghighirad, A. Makui, and G. A. Montazer, "Extension of fuzzy TOPSIS method based on interval-valued fuzzy sets," Applied Soft Computing Journal, vol. 9, no. 2, pp. 457461, 2009.

[23] S. Z. Guo and W. K. Yin, "Multiple attribute decision making method based on 2-type intuitionistic fuzzy information," Fuzzy Systems and Mathematics, vol. 27, no. 3, pp. 128-133, 2013.

[24] T.-Y. Chen, "An ELECTRE-based outranking method for multiple criteria group decision making using interval type-2 fuzzy sets," Information Sciences, vol. 263, pp. 1-21, 2014.

[25] T. Y. Chen, "An interval type-2 fuzzy PROMETHEE method using a likelihood-based outranking comparison approach," Information Fusion, vol. 25, pp. 105-120, 2015.

[26] X. H. Xu, Q. F. Wna, X. H. Chen, and Y. J. Zhou, "Study for a conflict measure for large group decision based on intervalvalued intuitionistic trapezoidal fuzzy number," Chinese Journal of Management Science, vol. 22, no. 8, pp. 115-122, 2014.

[27] N. Zamri and L. Abdullah, "A new linguistic variable in interval type-2 fuzzy entropy weight of a decision making method," Procedia Computer Science, vol. 24, pp. 42-53, 2013.

[28] P. Guo and H. Tanaka, "Decision making with interval probabilities," European Journal of Operational Research, vol. 203, no. 2, pp. 444-454, 2010.

[29] Z. Yue and Y. Jia, "An application of soft computing technique in group decision making under interval-valued intuitionistic fuzzy environment," Applied Soft Computing Journal, vol. 13, no. 5, pp. 2490-2503, 2013.

[30] L.-W. Lee and S.-M. Chen, "Fuzzy decision making based on likelihood-based comparison relations of hesitant fuzzy linguistic term sets and hesitant fuzzy linguistic operators," Information Sciences, vol. 294, pp. 513-529, 2015.

[31] L. A. Zadeh, "The concept of a linguistic variable and its application to approximate reasoning-I," Information Sciences, vol. 8, no. 3, pp. 199-249, 1975.

[32] P. Liu and F. Jin, "A multi-attribute group decision-making method based on weighted geometric aggregation operators of interval-valued trapezoidal fuzzy numbers," Applied Mathematical Modelling, vol. 36, no. 6, pp. 2498-2509, 2012.

[33] T.-Y. Chen, "A linear assignment method for multiple-criteria decision analysis with interval type-2 fuzzy sets," Applied Soft Computing Journal, vol. 13, no. 5, pp. 2735-2748, 2013.

[34] J.-Q. Wang and K.-J. Li, "Multi-criteria decision-making method based on intuitionistic normal fuzzy aggregation operators," System Engineering Theory \& Practice, vol. 33, no. 6, pp. 1501-1508, 2013. 


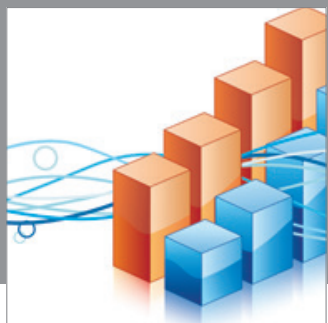

Advances in

Operations Research

mansans

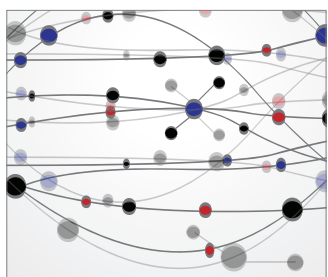

The Scientific World Journal
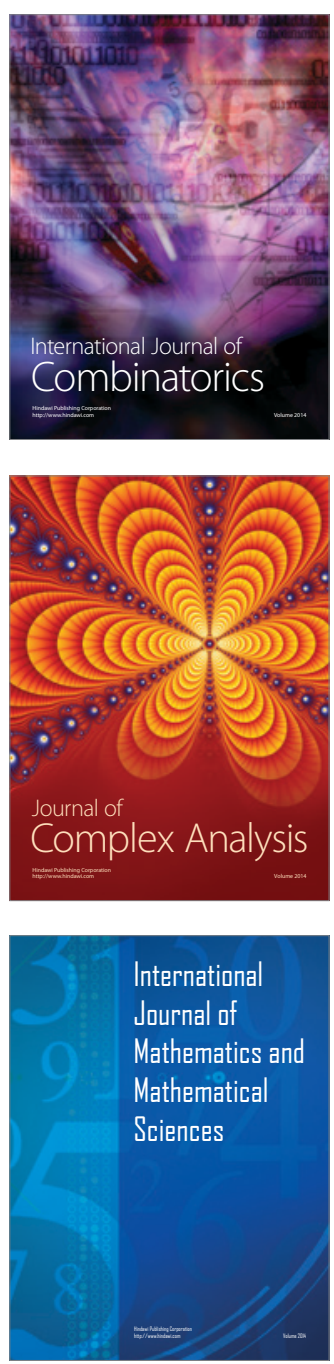
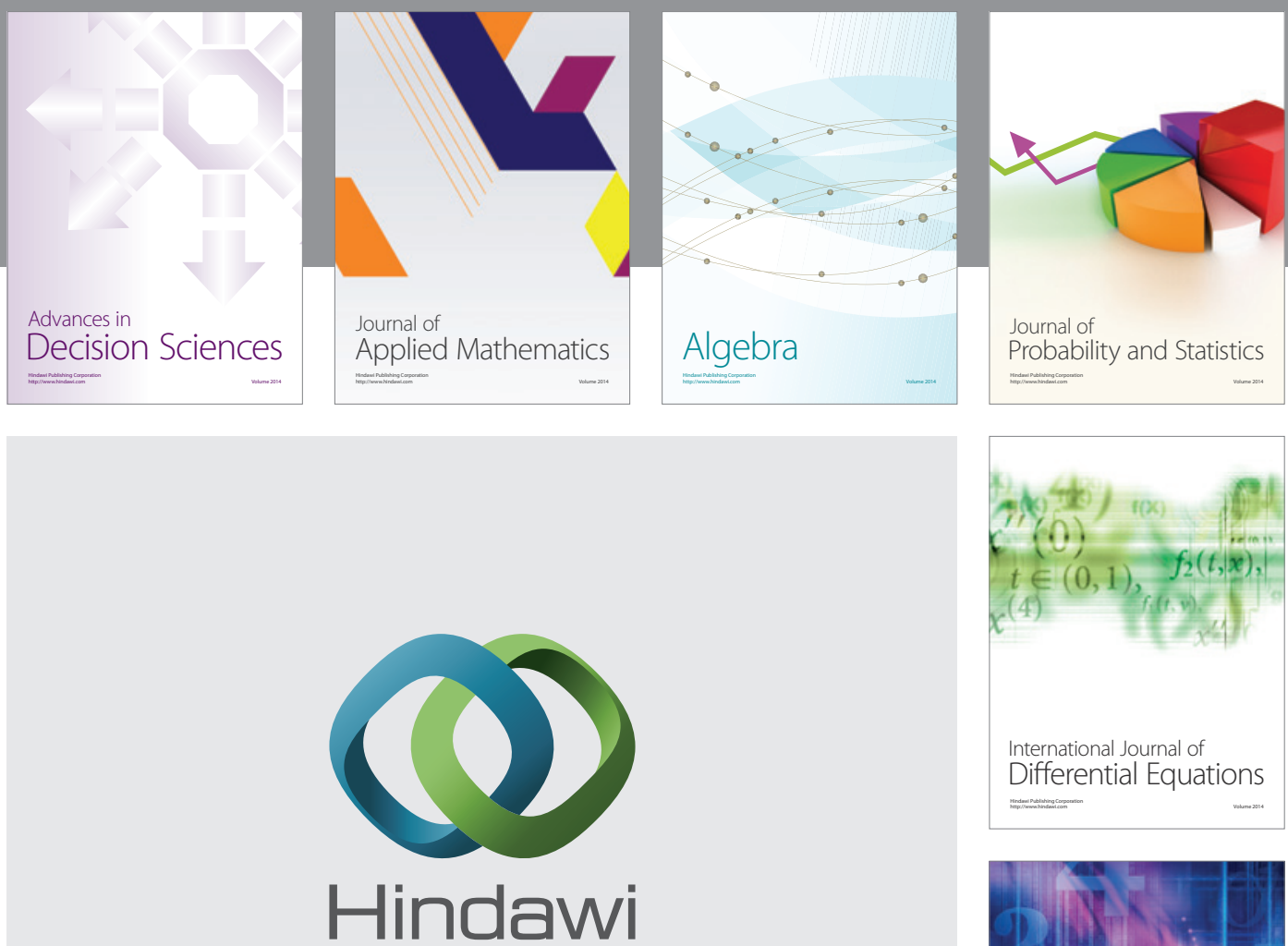

Submit your manuscripts at http://www.hindawi.com
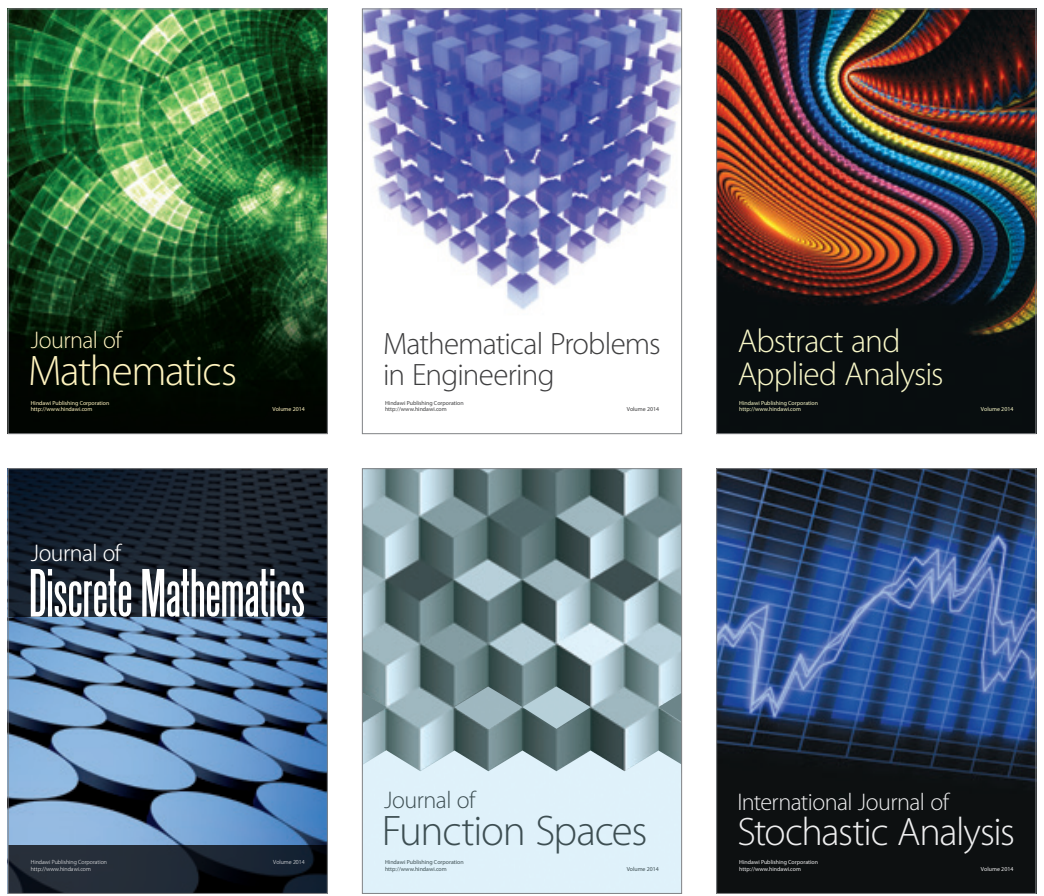

Journal of

Function Spaces

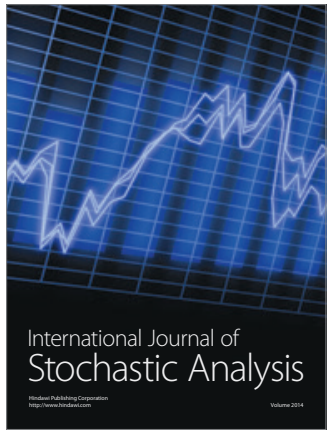

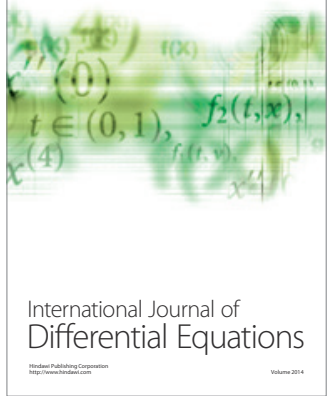
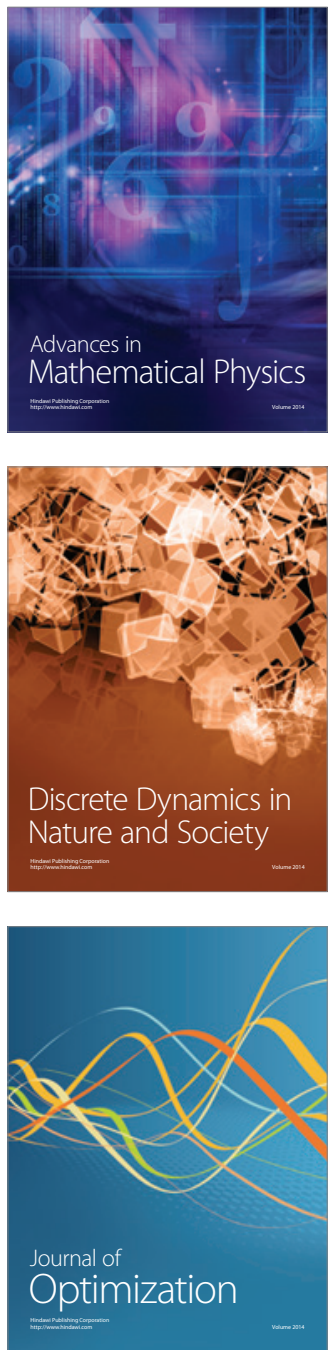